\title{
Simulation Modeling for Analysis of a (Q, r) Inventory System under Supply Disruption and Customer Differentiation with Partial Backordering
}

\author{
Parham Azimi, Mohammad Reza Ghanbari, and Hasan Mohammadi \\ Department of Industrial and Mechanical Engineenng, Qazvin Branch, Islamic Azad University, Barajin, Daneshgah St., Nokhbegan \\ Blvd., P.O. Box 34185141, Qazvin, Iran \\ Correspondence should be addressed to Parham Azimi, p.azimi@yahoo.com
}

Received 11 December 2011; Accepted 19 February 2012

Academic Editor: Azah Mohamed

Copyright () 2012 Parham Azimi et al. This is an open access article distributed under the Creative Commons Attribution License, which permits unrestricted use, distribution, and reproduction in any medium, provided the original work is properly cited.

We have modeled a new $(Q, r)$ inventory system which involves a single product, a supplier, and a retailer with customer differentiation under continuous review inventory policy. The supplier provides the retailer with all requirements, and the retailer sells products to the customers. The supplying process is randomly subject to disruptions. Partial backordering is applied when a stock out occurs, and customer can select either to leave the system without purchasing or to backorder products. The customers are categorized into two main classes regarding to their backordering probabilities. The main contribution of this paper is including the customer differentiation in the inventory model. We used simulation technique to verify the impact of supply disruptions and customer differentiation and carried out sensitivity analysis. To test the performance of the model, we have compared our model to one from the latest related research. As the results show, the average of total annual cost of the $(Q, r)$ inventory system is lower than that of the previously developed models such as $(r, T)$ inventory systems.

\section{Introduction}

Within the last years, inventory management has received wide attention in such a way the recent researches in this field have focused on the study of inventory system in the presence of supply chain problems such as supply disruption. Various factors can disrupt a supply chain system, including an equipment breakdown, a strike, bad weather, natural disasters, political instability, traffic interruptions, terrorism, and so on [1]. For example, on March 17, 2000, lighting hit a power line in Albuquerque, New Mexico, which in turn started a fire at a local plant owned by Royal Philips Electronics, damaging millions of microchips.

The representative literature of inventory management with respect to supply disruptions includes [2-4]. In general, the previous literature divides inventory systems into two categories. One is continuous-review-based-and another is periodic-review-based. Parlar [5] considers a continuousreview stochastic inventory problem with random demand and random lead time in the situation where supply may be disrupted. Gürler and Parlar [6] make further research contribution by considering an additional randomly available supplier in the problem that Parlar [5] addresses. ArreolaRisa and DeCroix [7] studied inventory management under random supply disruptions and partial backorders, with an $(s, S)$ policy being considered. Mohebbi $[8,9]$ assumes that the sales are lost when the stock is out. The references to periodic review aspect include $[10,11]$. Parlar et al. [10] analyze a finite-horizon periodic-review inventory model with backlogging. Samvedi and Jain [12] studied the impact of changes in the parameter values of periodic inventory policy on supply disruption situations. The process is simulated using discrete event simulation with the inventory and backorder levels taken as the output parameters. The study shows that there is a definite connection between the costs experienced at a level in the chain and its distance from the disruption point. Due to the difficulty of handling partial backorders, the inventory literature in this area 


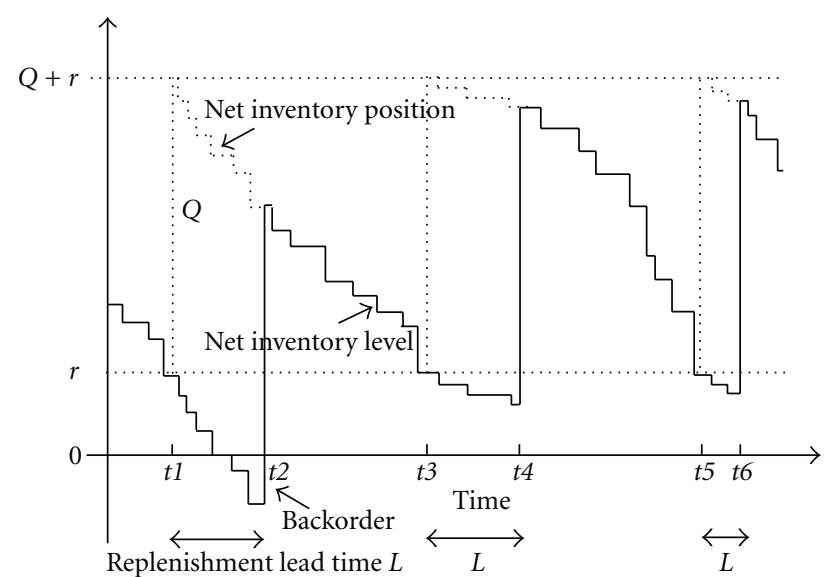

FIGURE 1: A standard $(Q, r)$ inventory policy without supply disruption.

is limited. Moinzadeh [13] considers a base-stock level inventory system with Poisson demand, constant resupply times, and partial backorders. Other inventory models with partial backorders can be found in Montgomery et al. [14], Kim and Park [15], and Posner and Yansouni [16]. Parlar and Berkin [17] study the classic EOQ problem with supply disruptions. Parlar and Perry [18] extend this analysis to a system, where orders may be placed before the inventory level reaches zero, and where there is a fixed cost for determining the state of the supplier. Weiss and Rosenthal [19] determine the optimal inventory policy when the timing (but not the duration) of supply disruptions is known in advance. To our knowledge, the only papers dealing with supply disruptions and random demand are $[2,5,16,17,20,21]$.

In this study, we consider a continuous-review inventory system in the presence of supply disruptions, with the relaxation of some assumptions that are made in the previous researches. For instance, we consider backordering in the stockout situations, which means that customers can choose to backorder unfulfilled products or not. The assumption relaxation makes the problem more realistic. In addition, customer differentiation which is included in the proposed model has not been considered in the previous continuousreview inventory systems. Due to the complexity of the problem, we have used simulation modeling to develop the mentioned inventory system. Furthermore, we investigate the impacts of supply disruptions and customer differentiation on the inventory system.

In Section 2, there is a description of the inventory policy that the retailer adopts and the considered problem. In Section 3, the process of simulation modeling for the concerned inventory system is explained. In Section 4, the simulation output is examined to determine the impacts of supply disruption and customer differentiation on the inventory system, and finally in Section 5, summary of the results and future opportunities are explained.

\section{Problem Description}

This paper considers a continuous-review inventory system with single product of a retailer, where supply may be disrupted. The supplier is not always available. The retailer sells products to the customers and replenishes the stock from its single supplier. When a supply disruption occurs, the supplier cannot fulfil the orders from the retailer. Only when the disruption issue is resolved can the orders be processed. We define the time period during which the supplier is available (i.e., under normal conditions) as its ON period, and the time period during which the supplier is not available (i.e., under disruption conditions) as its OFF period. ON and OFF periods represent the frequency and duration of supply disruptions, respectively. In other words, ON and OFF periods reflect the disruption severity of an unreliable supplier. The longer the ON periods, the less frequent the disruptions and the slighter the disruptions. On the contrary, the longer length of OFF periods, the longer the disruption duration and the more severe the disruptions. The standard $(Q, r)$ policies used when the supplier is available $(\mathrm{ON})$, that is, when the inventory position reaches the reorder point $r, Q$ units are ordered to raise the inventory position. The form of the policy changes when the supplier becomes unavailable (OFF) in which case orders cannot be placed when the reorder point $r$ is reached. However, as soon as the supplier becomes available again one orders enough to bring the inventory position up. In this paper, we use different combinations of mean values of $\mathrm{ON}$ and $\mathrm{OFF}$ periods to represent different supply disruption scenarios. In addition, in this paper replenishment lead time is deemed to be stochastic, which is consistent with the reality.

The retailer adopts a continuous-review inventory policy $(Q, r)$, where $r$ is reorder point (number of parts on hand when we placed an order), and $Q$ is reorder quantity. This policy means that, continuously, the retailer reviews its product inventory position and compares it with $r$ value and decides whether a replenishment is needed or not. When the inventory position is equal or less than $r$, the retailer orders $Q$ units of product. Figure 1 shows a standard $(Q, r)$ inventory policy that does not consider supply disruptions. Times $t 1$, $t 3$, and $t 5$ are the points where inventory position reaches the $r$, and an order is placed to the supplier. $t 2, t 4$, and $t 6$ are time points when the ordered products are received by the retailer. Time periods $t 2-t 1, t 4-t 3$, and $t 6-t 5$ are three realizations of stochastic replenishment lead time $L$.

Figure 2 shows an $(Q, r)$ inventory system where supply disruptions are taken into account. As seen in this figure, the red line segments on time axes represent OFF periods of the supplier, and other line segments on the axes represent ON periods. Similar to those in Figure 1 , time points $t 1$, $t 3$, and $t 5$ are three reorder points. However, the orders placed at these time points receive different treatments. At time points $t 1$ and $t 3$, the supplier is available (i.e., in ON periods), and the orders are processed and shipped out immediately. The retailer receives the products at time points $t 2$ and $t 4$, respectively. However, at time point $t 5$, the supplier is in an OFF period (i.e., under disruption status), so the order cannot be processed until the supplier restores to its normal status. Hence, the order is processed and shipped out after the OFF period ends, which occurs at time point $t 6$. The retailer finally receives the products at time point $t 7$. Similarly, time periods $t 2-t 1, t 4-t 3$, and $t 7-t 5$ 


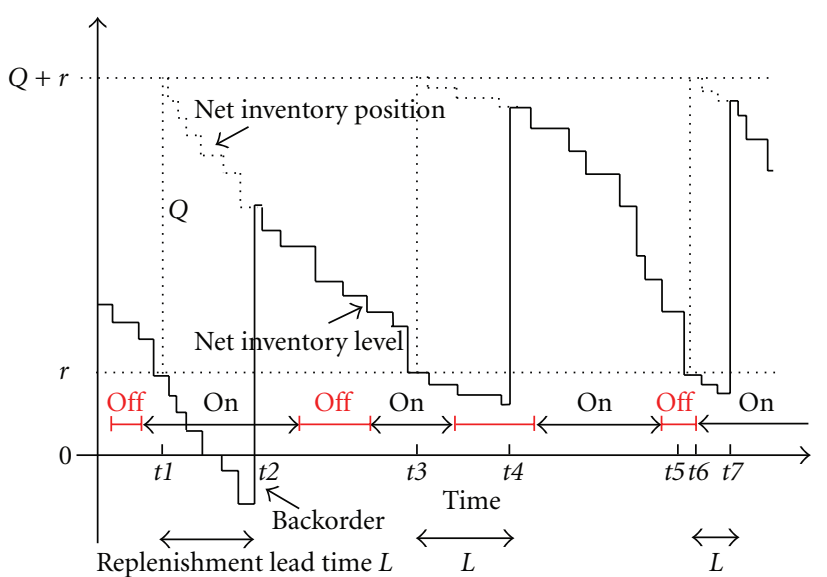

FIGURE 2: A $(Q, r)$ inventory policy with supply disruption.

are three realizations of stochastic replenishment lead time $L$. Comparing Figure 2 with Figure 1, it is obvious that supply disruptions delay order replenishments.

In this paper, we study partial backordering in the stockout situations. When the retailer is out of stock, a customer may choose to backorder the products he/she needs or to abandon the purchase order and leave for other sellers (i.e., lost sale). The retailer incurs backorder cost or lost sale cost accordingly. In general, unit backorder cost per time unit is less than unit lost sale cost, since the retailer may obtain profits from selling backorders.

In addition, we allow the number of outstanding orders to be more than one. We also incorporate customer differentiation in the discussed inventory system. Customers are segmented based on their backorder probabilities in the stockout situations and are differentiated into two classes. One class has higher backorder probability, while the other class has lower backorder probability. For convenience, these two classes are denoted by classes I and II, respectively. To acknowledge class I for their higher backorder probability, the retailer provides them with high priority to receive backorders.

All the above considerations, combined with the complex nature of a continuous-review inventory system, make it very difficult to study this inventory management problem by using an analytical method. In this paper, we will utilize simulation techniques [22] to investigate the concerned inventory system. The used measure for performance is the average annual total cost of the retailer, which includes annual ordering cost, annual inventory holding cost, annual backorder cost, and annual lost sale cost. The following is the to-be-used notations [23] (see Nomenclature).

The calculation formulas of the annual ordering cost, annual holding cost, annual backorder cost, and annual lost sale cost from each customer class are as follows:

AOC $=s *$ number of ordering during $T$

$\mathrm{AHC}=\int_{0}^{T} h * \max (\mathrm{IL}(t), 0) d t$,

$$
\begin{aligned}
& \mathrm{ABC}_{i}=\int_{0}^{T} b_{i} * \mathrm{BoQ}_{i}(t) d t, \quad i \in\{\mathrm{I}, \mathrm{II}\} \\
& \mathrm{ALC}_{i}=l_{i} * \mathrm{NLS}_{i}, \quad i \in\{\mathrm{I}, \mathrm{II}\}
\end{aligned}
$$

Therefore, the annual total cost of the retailer (i.e., the sum of the above costs) is

$$
\begin{aligned}
\mathrm{ATC} & =\mathrm{AOC}+\mathrm{AHC}+\mathrm{ATBC}+\mathrm{ATLC} \\
& =\mathrm{AOC}+\mathrm{AHC}+\sum_{i \in\{\mathrm{I}, \mathrm{II}\}} \mathrm{ABC}_{i}+\sum_{i \in\{\mathrm{II}, \mathrm{II}\}} \mathrm{ALC}_{i} .
\end{aligned}
$$

\section{The Simulation Model}

The structure of the model is made up of two subsystems: customer demand subsystem and inventory replenishment subsystem, as shown in Figures 3 and 4, respectively. The two subsystems can be realized by using Enterprise Dynamics 8 (ED. 8) simulation software.

3.1. Customer Demand Subsystem. Figure 3 describes the customer demand subsystem. When a customer arrives, the retailer checks its net inventory level. There exist three situations based on net inventory level. The first situation is that the net inventory level is positive, and there is enough stock to satisfy the demand of the customer. Under this situation, the customer purchases the products with satisfaction. The retailer then updates its net inventory level and inventory position accordingly.

The second situation is that the net inventory level is positive, but there is no enough stock for the customer's demand. Under this situation, the customer takes all available products and decides whether to backorder the unfulfilled products or not. The third situation is that the net inventory level is nonpositive, and there are no products available at all. Under this situation, the customer can either backorder the unfulfilled products or leave without ordering. In the second and third situations, we differentiate customers since different customer classes have different backorder probabilities.

If a customer chooses to backorder, the net inventory level and inventory position of the retailer decrease by the demand size of the customer. Besides, the backorder quantity of the corresponding customer class is equal to minus net inventory level if it is the second situation or increases by the demand size of the customer if it is the third situation. Now consider the other case. If the customer chooses not to backorder, part of the sale is lost when it is the second situation or the entire sale is lost when it is the third situation. The resulting lost sale cost is calculated accordingly, as shown in Figure 3. Note that the number of lost sale cost is the demand size of the customer minus current net inventory level in the second situation, and it is equal to the demand size of the customer in the third situation. In addition, if it is the second situation, the inventory position decreases by the current net inventory level which is the quantity of all 


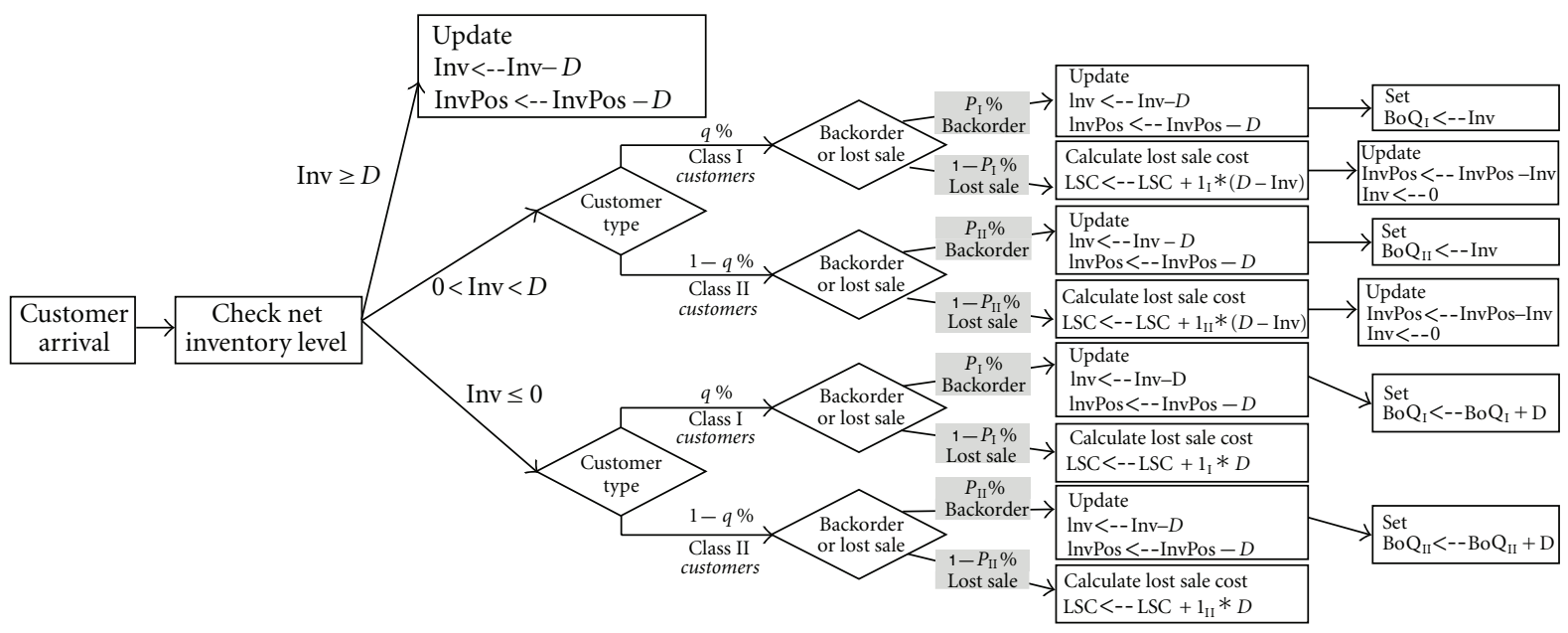

Notations:

Inv: net inventory level

li: unit lost sale cost for class I customers

InvPos: inventory position

BoQi: backorder quantity of class I customers

D: demand size of a customer

LSC: lost sale cost

$a<-$ b: set the value of $a$ to be equal to $b$

Figure 3: Customer demand subsystem.

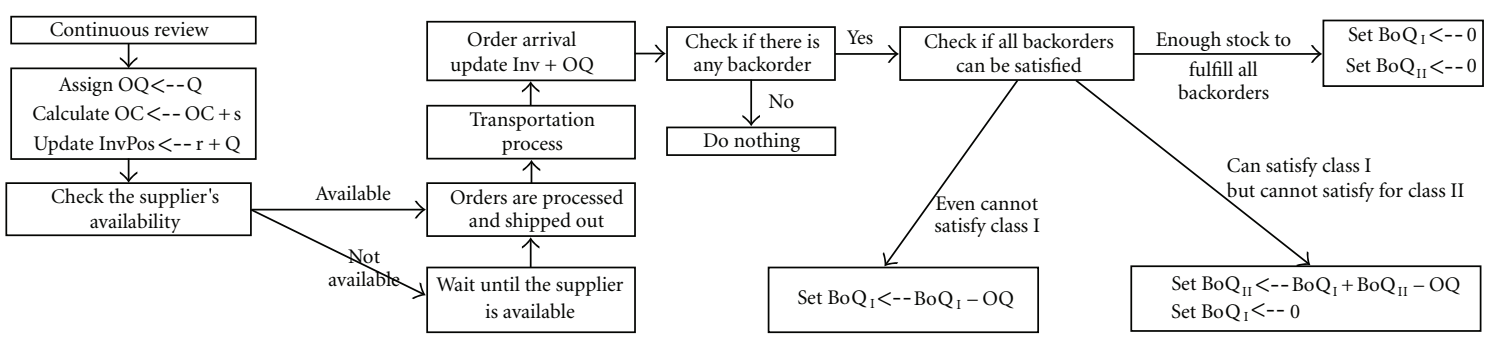

Notations:

Inv: net inventory level

InvPos: inventory position

$s$ : setup cost for each order placement

OQ: order quantity
OC: ordering cost

BoQi: backorder quantity from customer class I

$\mathrm{a}<--\mathrm{b}$ : set the value of $\mathrm{a}$ to be equal to $\mathrm{b}$

FIGURE 4: Inventory replenishment subsystem.

products on stock. The net inventory level is subsequently set to be zero, since the customer takes all available products [23].

3.2. Inventory Replenishment Subsystem. Figure 4 describes the inventory replenishment subsystem. Continuously, the retailer reviews its inventory position and determines whether replenishment is needed or not. According to the adopted $(Q, r)$ inventory policy, the inventory position needs to be increased to $r+Q$. Therefore, the needed product quantity (i.e., order quantity) is equal to $Q$. The corresponding ordering cost is then calculated as shown in Figure 4. When an order is placed, the availability of the supplier needs to be checked. If the supplier is in its normal condition, it processes the order immediately. If the supplier encounters a disruption at that time, the order has to wait for being processed until the supplier restores to its normal status. After being processed, the order is shipped out. Going through the transportation process, the order arrives at the retailer, and the net inventory level is increased correspondingly. If there exist unfulfilled backorders, the retailer needs to fulfil them. Three situations need to be considered. The first situation is that the stock is enough for all backorders to be fulfilled. Mathematically, that is, $\mathrm{OQ} \geq$ $\mathrm{BoQ}_{\mathrm{I}}+\mathrm{BoQ}_{\mathrm{II}}$, where $\mathrm{OQ}$ and $\mathrm{BoQ}_{i}(i=\mathrm{I}$; II $)$ represent order quantity and backorder quantity from customer class $i$, respectively. The second situation is that the backorders from customer class I can be satisfied but only part of the backorders from customer class II can be satisfied. That is, 


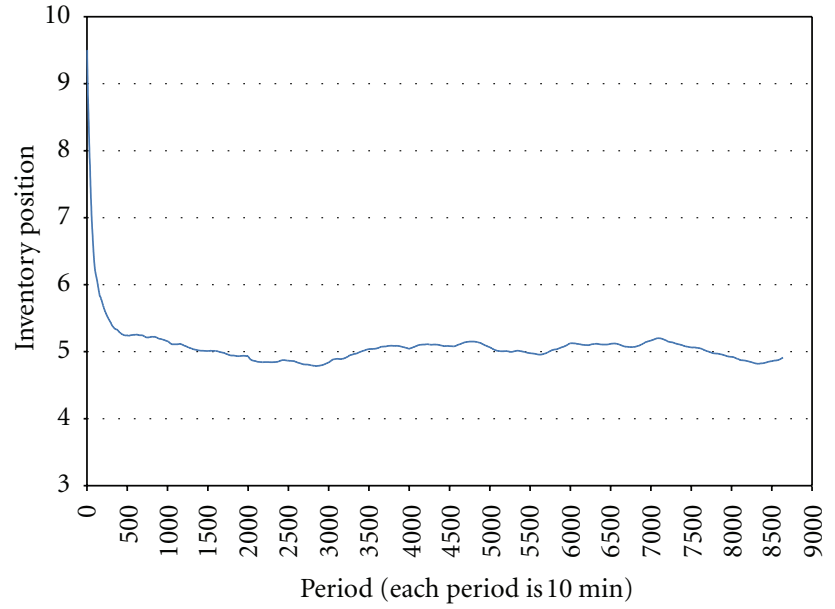

FIGURE 5: Determining warmup period.

Table 1: Empirical distribution of customer's demand size.

\begin{tabular}{lccccc}
\hline Demand size & 1 & 2 & 3 & 4 & 5 \\
Probability & 0.1 & 0.25 & 0.3 & 0.25 & 0.1 \\
\hline
\end{tabular}

$\mathrm{BoQ}_{\mathrm{I}} \leq \mathrm{OQ}<\mathrm{BoQ}_{\mathrm{I}}+\mathrm{BoQ}_{\mathrm{II}}$. The third situation is that the arriving products even cannot satisfy the backorders of customer class I (i.e., $\mathrm{OQ}<\mathrm{BoQ}_{\mathrm{I}}$ ) [23].

3.3. The Input Data of the Model. In this section, we present the input data of the model. The customer demand of the retailer is assumed to follow an empirical distribution, where customer interarrival time follows an exponential distribution with a mean of $\lambda=0.2$ days, and each customer's demand size $D$ has a probability distribution as shown in Table 1. Without loss of reasonability, we make the following assumptions. The setup cost for each order placement is $s=\$ 10$; unit product cost is $c=\$ 10$; unit holding cost per time unit is $h=\$ 2$; unit backorder costs per time unit from customer classes I and II are $b_{\mathrm{I}}=\$ 1.8$ and $b_{\mathrm{II}}=\$ 1.5$, respectively; unit lost sale costs from customer classes I and II are $l_{\mathrm{I}}=\$ 4$ and $l_{\mathrm{II}}=$ $\$ 3$, respectively. The supplier's ON and OFF periods are supposed to follow exponential distributions, with means being $u$ days and $v$ days, respectively. Note that $u$ and $v$ are used to represent the magnitude of supply disruptions. The transportation duration of an order is assumed to follow a normal distribution with a mean of 4 days and a standard deviation of 0.5 . The initial net inventory level and inventory position of the retailer are arbitrarily set to be $\mathrm{IL}_{0}=\mathrm{IP}_{0}=10$. Such settings prevent the initial inventory status from being unrealistically "empty and idle." Later we will warmup the simulation model to remove the influences that the initial settings bring about. The inventory policy parameters $r$ and $Q$ are decision variables. Their values are to be determined by the experiments.

3.4. Warmup Period. In the beginning of the simulation, the model is empty without any inventory. Therefore, the
TABLE 2: The design for the first experiment.

\begin{tabular}{lc}
\hline Parameter & Values (units: days) \\
\hline$u$ & $20 ; 60 ; 120$ \\
$v$ & $1 ; 5 ; 10$ \\
\hline
\end{tabular}

data obtained from that may not be appropriate criteria for analysis. To avoid this matter, a period of time is taken into account for the model as the warmup period. In this study we have used the Welch method [11]. The index we have used here is the average of the inventory position. By drawing the graphic diagram of the moving average of the index calculated it was determined that after period 1500 (each period is 10 minutes), the model shows a stable behaviour against the index under consideration. Therefore, in the analysis of the model we will suppose $1500 * 10$ minutes $=$ 15000 minutes for the warmup period. Figure 5 determines that the system becomes stable after period 1500 .

\section{Experiment and Simulation Result Analysis}

In this section, we simulate the inventory system according to the model described in Sections 2 and 3. We also conduct experiments for investigating the impacts of supply disruptions and customer differentiation on the inventory system. We design several scenarios for the experiments. For each scenario, the optimal inventory policy $(Q, r)$ and the corresponding minimum average annual total cost are obtained. Because the goal of the retailer is to minimize its annual total cost, the minimum average annual total cost is taken as performance measure for each scenario. We then examine the obtained minimum average annual total costs from the experiments, expecting to discover the influences of the above two factors on the inventory system and to obtain some managerial insights for the retailer.

4.1. Experimental Design and Simulation Settings. To investigate the impact of supply disruptions on the inventory system, we conduct the following experiment. We reasonably assume that $10 \%$ of customers belong to class I, and that when a stockout occurs, $80 \%$ of customer class I and $10 \%$ of customer class II choose to backorder, that is, $q=10 \%$; $p_{\mathrm{I}}=80 \% ; p_{\mathrm{II}}=10 \%$. We use $u$ and $v$ to construct different scenarios of supply disruptions, as listed in Table 2. Since $u$ represents the frequency of supply disruptions, three values of $u$ denote severe, moderate, and slight disruptions, respectively. Similarly, as the indicator of supply disruption duration, three values of $v$ denote slight, moderate, and severe supply disruptions, respectively. We combine $u$ and $v$ and generate 9 scenarios. For each scenario, we utilize simulation techniques to obtain optimal reorder point $r^{*}$ and order quantity $Q^{*}$, so that such an inventory policy can lead to the minimum average annual total cost of the retailer.

On the other hand, when looking into the impact of customer differentiation on the inventory system, we consider two cases of supply disruptions for the sake of completeness: $\{u=60 ; v=1\}$ and $\{u=60 ; v=10\}$. 
TABLE 3: The design for the second experiment.

\begin{tabular}{lc}
\hline Parameter & Values \\
\hline$q$ & $5 \% ; 10 \% ; 20 \% ; 40 \%$ \\
$p_{\text {I }}$ & $60 \% ; 90 \%$ \\
$p_{\text {II }}$ & $5 \% ; 20 \%$ \\
\hline
\end{tabular}

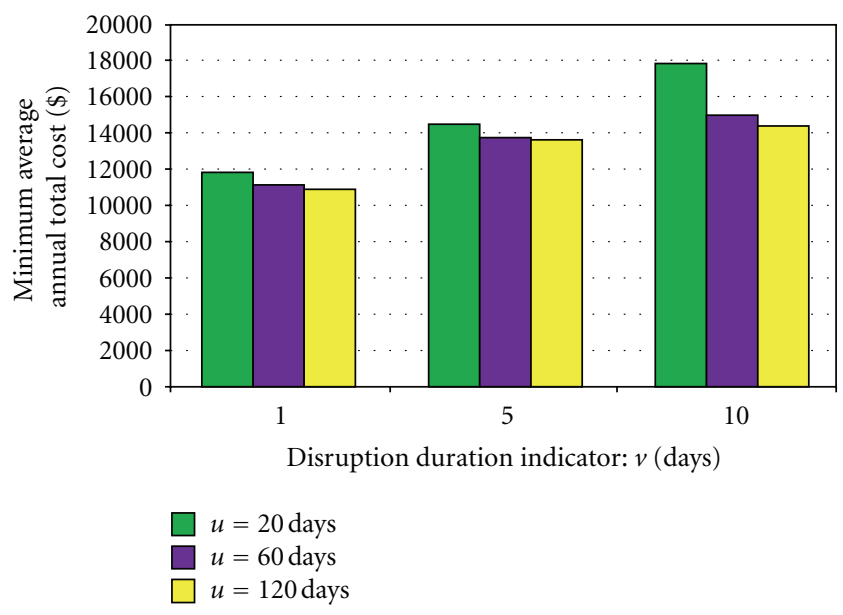

Figure 6: The impact of $u$ on ATC under different scenarios of $v$.

Under each case, we take into account different scenarios of customer differentiation as shown in Table 3 . The reason we do not consider bigger values for $q$ (e.g., $60 \%$ and $80 \%$ ) is that, in real life the customers who would choose to backorder only take a small fraction and not to mention the fraction of customer class I who have large probability to backorder products. In addition, based on the definitions, $p_{\text {I }}$ would take bigger values, and $p_{\text {II }}$ would take smaller values. We consider two values for $p_{\mathrm{I}}$ and $p_{\mathrm{II}}$, respectively. Totally in this experiment, for each supply disruption case, 16 scenarios of customer differentiation are studied. As in the above experiment regarding supply disruptions' impact, for each scenario, optimal reorder point $r^{*}$ and order quantity $Q^{*}$ are obtained for the minimum average annual total cost of the retailer.

As we showed in Section 3.4, the warmup period is obtained to be equal to 15000 minutes. In the experiments, furthermore, for each scenario in each experiment, we utilize the optimization tool Opt-Quest in the ED simulation software to obtain the optimal inventory policy $\left(Q^{*}, r^{*}\right)$ and the minimum average annual total cost. When conducting optimization, for each possible combination of $(Q, r)$, we run $n=10$ replications. For each combination of $(Q, r)$, the average annual total cost of the retailer is derived from the data generated from 10 replications. For each scenario in each experiment, the obtained minimum average annual total cost is recorded as the result.

4.2. Results and Analysis. The experimental results are illustrated in Figures 6-9. Figure 6 shows the impact of disruption frequency indicator $u$ on the minimum average annual total cost, based on different scenarios of disruption

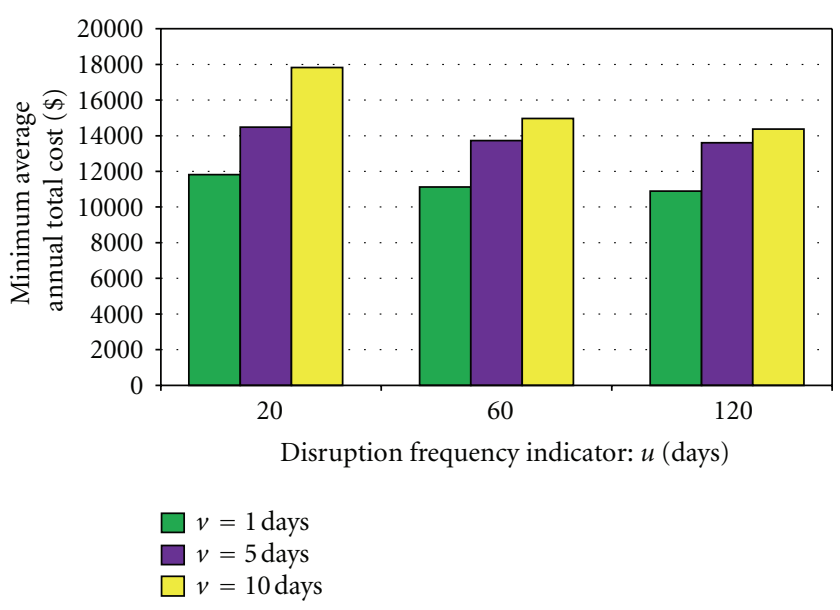

FIgURE 7: The impact of $v$ on ATC under different scenarios of $u$.

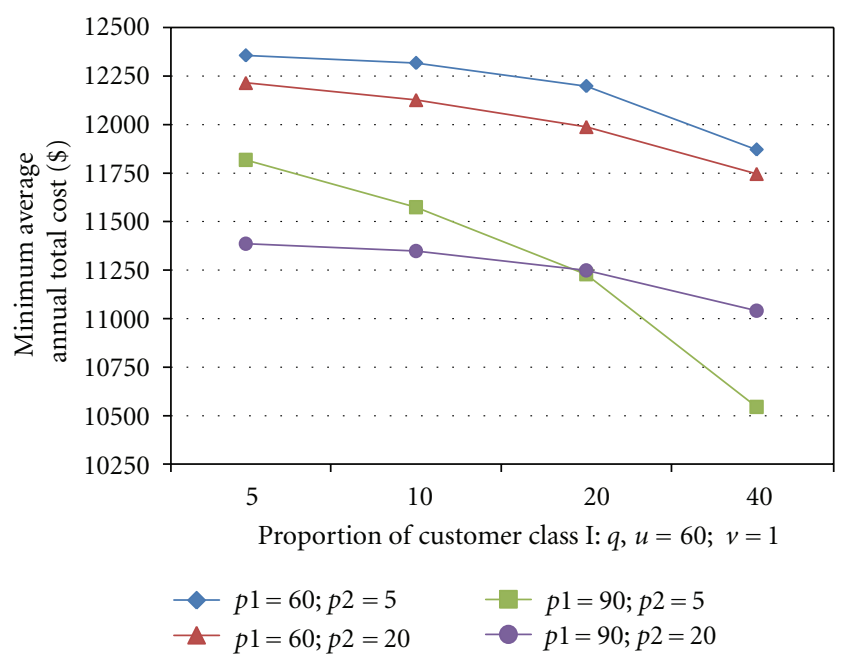

Figure 8: The impact of customer differentiation on ATC under the disruption scenario of $\{u=60 ; v=l\}$.

duration indicator $v$. We can see that, for each $v$, the minimum average annual total cost of the retailer decreases in $u$. Moreover, the decrease magnitude increases in $v$. This figure indicates that, given a fixed mean value of disruption duration (i.e., $v$ ); less frequent disruptions (i.e., bigger $u$ ) lead to smaller minimum average annual total cost. Figure 6 also implies that, when disruption duration is short, there are no big differences regarding the impacts of different disruption frequency values on the minimum average annual total cost. The differences are more significant when disruption duration is longer.

Figure 7 illustrates the impact of disruption duration indicator $v$ on the minimum average annual total cost, based on different scenarios of disruption frequency indicator $u$. It is obvious that, for each $u$, the minimum average annual total cost of the retailer increases in $v$. Moreover, the increase magnitude decreases in $u$. This figure reveals that, when disruption frequency is given, shorter disruption 


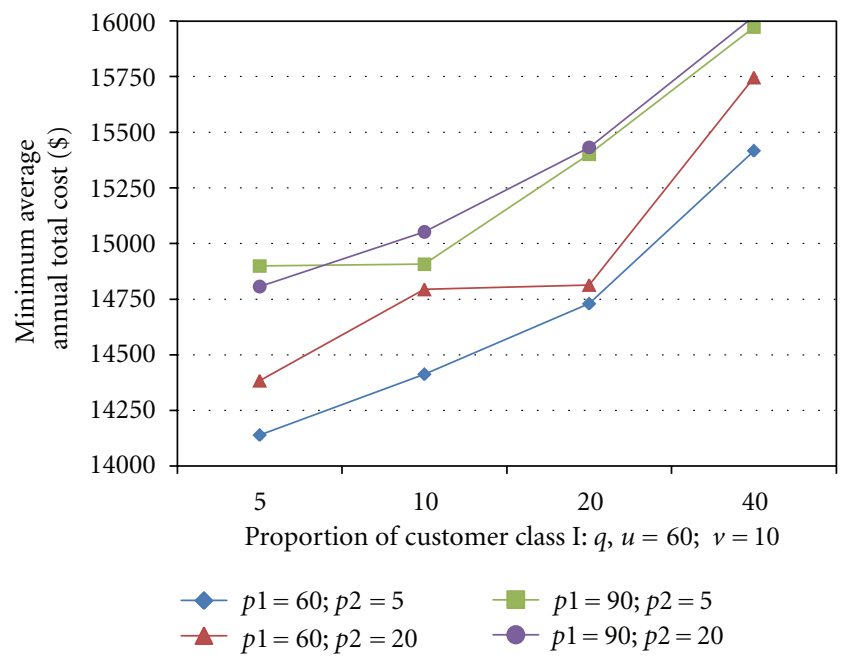

FIGURE 9: The impact of customer differentiation on ATC under the disruption scenario of $\{u=60 ; v=10\}$.

duration leads to smaller minimum average annual total cost. Figure 7 also shows that, the impacts of different disruption duration values on the minimum average annual total cost differ significantly when disruption frequency is large (i.e., small $u$ ). The differences become smaller when disruption frequency gets smaller (i.e., larger $u$ ).

Figures 6 and 7 tell the retailer that, two supply disruption magnitude indicators $u$ and $v$ play important roles in selecting a supplier. In the real world, a supplier is more or less subject to supply disruptions. A supplier with less frequent disruptions (i.e., bigger $u$ ) or shorter disruption duration (i.e., smaller $v$ ) is a better choice for the retailer. In addition, assume that a group of suppliers are to be selected. If these suppliers have the same disruption frequency that is little enough, then there are no big differences among the selection of these suppliers even if their disruption duration values differ dramatically. Similarly, if these suppliers have the same disruption duration that is short enough, then there are no big differences among the selection of these suppliers even if their disruption frequency values differ remarkably.

Figure 8 demonstrates the impact of customer differentiation on the minimum average annual total cost under the disruption scenario of $\{u=60 ; v=1\}$. As stated in Section 4.1, four possible $q$ values are considered. For each $q$ value, four scenarios of the combination of $p_{\mathrm{I}}$ and $p_{\mathrm{II}}$ are investigated. Figure 8 shows that, whatever scenario of the combination of $p_{\mathrm{I}}$ and $p_{\mathrm{II}}$, the minimum average annual total cost decreases in $q$. This implies that, when the supply disruption scenario is $\{u=60 ; v=1\}$, the more customers from class $I$, the smaller the minimum average annual total cost of the retailer. Therefore, the retailer should attract more customers to join class I. Furthermore, from Figure 8 we can find that, when $q$ and $p_{\text {I }}$ are fixed, the minimum average annual total cost in the case of $p_{\mathrm{II}}=20 \%$ is smaller than that in the case of $p_{\mathrm{II}}=5 \%$, except the case for the situation of $p_{\mathrm{I}}$ $=90 \%, p_{\mathrm{II}}=5 \%$ and, $q=40 \%$. Besides, when $q$ and $p_{\mathrm{II}}$ are fixed, the minimum average annual total cost in the case of
$p_{\mathrm{I}}=90 \%$ is smaller than that in the case of $p_{\mathrm{I}}=60 \%$. Both findings imply that when the proportion of customer class I and the backorder probability of the one class are fixed, the more the customers from the other class who choose to backorder in the stockout situations, the less the minimum average annual total cost. Figure 8 also reveals that when $p_{\text {I }}=60 \%$, whatever $p_{\text {II }}$ value, the decrease magnitude of the minimum average annual total cost in $q$ is slight. However, when $p_{\mathrm{I}}=90 \%$, the decrease magnitude is large. This reflects that the larger the backorder probability of class I, the more significant the decrease of the minimum average annual total cost with the increase of the proportion of class I.

Figure 9 exhibits the impact of customer differentiation on the minimum average annual total cost under the disruption scenario of $\{u=60 ; v=10\}$. Likewise, four $q$ values and four scenarios of the combination of $p_{\mathrm{I}}$ and $p_{\text {II }}$ are considered. Figure 9 shows that whatever scenario of the combination of $p_{\mathrm{I}}$ and $p_{\mathrm{II}}$, the minimum average annual total cost increases in $q$. This implies that when the supply disruption scenario is $\{u=60 ; v=10\}$, the less the customers from class I and the smaller the minimum average annual total cost of the retailer. Therefore, the retailer should reduce the number of class I customers. This is totally contrary to the above situation, where the supply disruption scenario is $\{u=60 ; v=1\}$. This indicates the influence of supply disruptions on customer differentiation's impact on the inventory system. Figure 9 also shows that when $q$ and $p_{\text {I }}$ are fixed, the minimum average annual total cost in the case of $p_{\mathrm{II}}=5 \%$ is smaller than that in the case of $p_{\mathrm{II}}=20 \%$. This is not the case for the situation of $p_{\mathrm{I}}=90 \%$ and $q=$ $5 \%$. Besides, when $q$ and $p_{\text {II }}$ are fixed, the minimum average annual total cost in the case of $p_{I}=60 \%$ is smaller than that in the case of $p_{\mathrm{I}}=90 \%$. These observations indicate that for the retailer, when the proportion of customer class I is fixed, for either value of $p_{\mathrm{II}}$, smaller backorder probability of customer class I leads to smaller minimum average annual total cost. In addition, when the proportion of customer class I is fixed and $p_{\mathrm{II}}=5 \%$, smaller backorder probability of customer class I leads to smaller minimum average annual total cost.

Now, we want to compare the results of the experiments to the one developed by $\mathrm{Li}$ and Chen [23]. The only difference is that they have used periodic-review model, while here we have developed a continuous-review model. First of all, the supply disruptions have been compared between the two models. In Table 4 and Figure 10, the average annual costs of the two models have been shown for different values of $u$ and $v$. For each scenario, the model has been replicated 30 times, and the averages have been listed in the table.

As the results show, the continuous-review model dominates the other model in all scenarios with different values of $u$ and $v$ ( $P$ value is 0.0001$)$. The main reason is that the backorders and the lost sales costs are lower in the continuous model. Now, we compare the two models based on backorder and lost sales rates. The results have been summarized in Table 5 and Figure 11.

As the results show, the average annual costs (ATC) of the continuous-review model is lower that the periodic one in all scenarios ( $P$ value is 0.0001$)$. 
TABLE 4: Compare current model with previous study under different scenarios of $v$ and $u$.

\begin{tabular}{|c|c|c|c|c|c|c|c|}
\hline \multirow{2}{*}{ Scenario number } & \multirow{2}{*}{$Q$} & \multirow{2}{*}{$p 1$} & \multirow{2}{*}{$p^{2}$} & \multicolumn{2}{|c|}{ Disruption } & \multicolumn{2}{|c|}{ Average annual total cost } \\
\hline & & & & $u$ & $v$ & Current model $(Q, r)$ & Previous model $(r, T)$ \\
\hline 1 & & & & 120 & 1 & 10891 & 13900 \\
\hline 2 & & & & 60 & 1 & 11127 & 13900 \\
\hline 3 & & & & 20 & 1 & 11813 & 13950 \\
\hline 4 & & & & 120 & 5 & 13608 & 14150 \\
\hline 5 & 10 & 80 & 10 & 60 & 5 & 13731 & 14400 \\
\hline 6 & & & & 120 & 10 & 14372 & 14800 \\
\hline 7 & & & & 20 & 5 & 14479 & 15750 \\
\hline 8 & & & & 60 & 10 & 14973 & 15800 \\
\hline 9 & & & & 20 & 10 & 17826 & 18700 \\
\hline
\end{tabular}

TABLE 5: Compare current model with previous study under impact of customer differentiation on the inventory system.

\begin{tabular}{|c|c|c|c|c|c|c|c|}
\hline \multirow{2}{*}{ Scenario number } & \multirow{2}{*}{$U$} & \multirow{2}{*}{$v$} & \multirow{2}{*}{$q$} & \multirow{2}{*}{$P 1$} & \multirow{2}{*}{$P 2$} & \multicolumn{2}{|c|}{ Average annual total cost } \\
\hline & & & & & & Current model $(Q, r)$ & Previous model $(r, T)$ \\
\hline 1 & & & 40 & 90 & 5 & 10545 & 13670 \\
\hline 2 & & & 40 & 90 & 20 & 11041 & 13050 \\
\hline 3 & & & 20 & 90 & 5 & 11228 & 13670 \\
\hline 4 & & & 20 & 90 & 20 & 11249 & 13270 \\
\hline 5 & & & 10 & 90 & 20 & 11348 & 13450 \\
\hline 6 & & & 5 & 90 & 20 & 11386 & 13600 \\
\hline 7 & & & 10 & 90 & 5 & 11574 & 13500 \\
\hline 8 & 60 & 1 & 40 & 60 & 20 & 11746 & 13370 \\
\hline 9 & & & 5 & 90 & 5 & 11818 & 13700 \\
\hline 10 & & & 40 & 60 & 5 & 11871 & 14050 \\
\hline 11 & & & 20 & 60 & 20 & 11988 & 13800 \\
\hline 12 & & & 10 & 60 & 20 & 12127 & 14050 \\
\hline 13 & & & 20 & 60 & 5 & 12198 & 14150 \\
\hline 14 & & & 5 & 60 & 20 & 12215 & 14200 \\
\hline 15 & & & 10 & 60 & 5 & 12317 & 14250 \\
\hline 16 & & & 5 & 60 & 5 & 12356 & 14300 \\
\hline
\end{tabular}

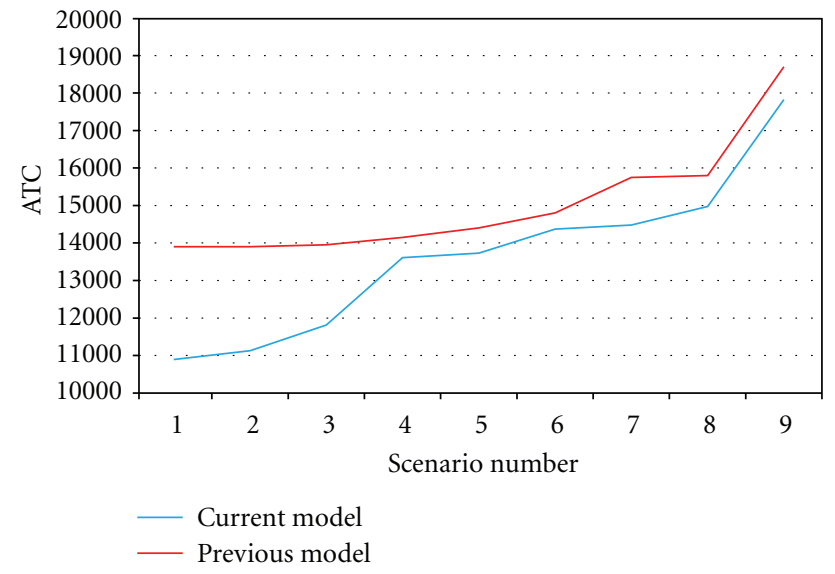

Figure 10: Compare current model with previous study under different scenarios of $v$ and $u$.

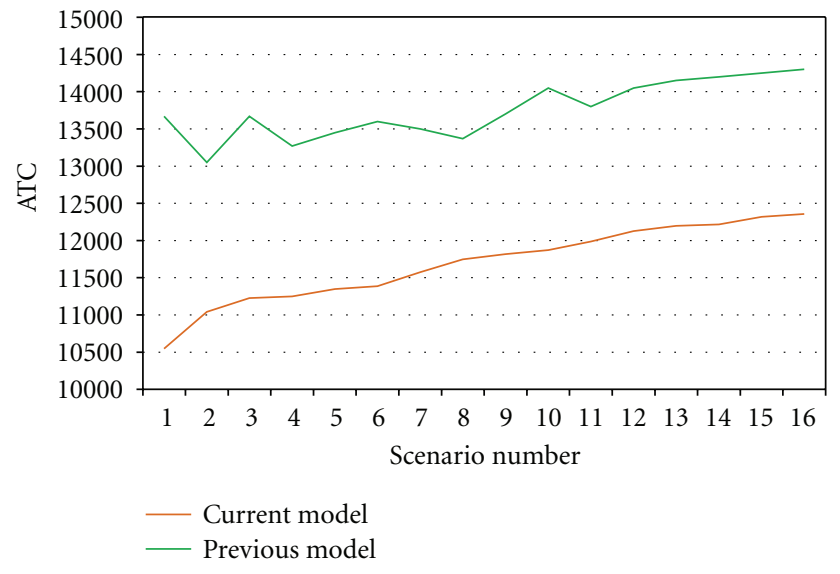

Figure 11: Compare current model with previous study under impact of customer differentiation on the inventory system. 


\section{Conclusions}

In the paper, a continuous-review inventory model $(Q, r)$ with supply disruption and customer differentiation has been studied. We have considered a single supplier who may have some disruptions in its supply, one retailer who puts the orders to the supplier, and some customers for products. The first contribution of the current study is that we have considered a right for the customer to suspend the backorders or cancel them, while in majority of past studies just one of the cases has been considered, and we allowed the system to have more than one order in the pipeline, which made our model to be more realistic. For studying the inventory system, we used a simulation model consisting of two subsystems including demand subsystem and replenishment subsystem; then the effects of supply disruptions and customer differentiations on average annual costs of the inventory system. Simulation is a very powerful technique for such a complicated system where using common techniques such as mathematical programming models is very difficult. Several experiments have been carried out to optimize the system for setting the best strategies for the retailer. The results show that the both underlying factors $(u$ and $v)$ have significant effects on selecting the supplier. The retailer must select a supplier with low degree of frequency and duration of disruptions. If one of these factors is low, the other factor has insignificant effect on supplier selection. The other result is that the effect of customer differentiation depends on supply disruptions. When the frequency of disruptions is normal and the duration is low, the average annual costs will minimize by selecting the high-priority customers who may have the high level of probability for their backorders, and the ATC will decrease if the probability of backorders increases for both classes of customers. When the frequency of disruptions is normal and the duration increases, the situation changes. In this case, the ATC increases when the amount of high level customers increases and if the probability of backorders is low for both classes, the ATC decreases.

We have also shown that the ATC of a continuousreview model is significantly lower than a periodic one which is taken from a latest research. For future studies, we recommend to consider the price factor in the model as well. The normal fluctuation on product prices may have a great effect on the inventory system costs.

\section{Decision Variables}

Q: Order quantity

$r$ : Reorder point.

Objective Function

ATC: Annual total cost.

\section{Other Parameters and Notations}

$D$ : Stochastic demand size of a customer $\lambda: \quad$ The mean interarrival time of customers

$L: \quad$ Replenishment lead time

T: $\quad$ Time horizon as one year, that is, 365 days

$\mathrm{IL}_{0}$ : The initial net inventory level of the retailer

$\operatorname{IL}(t)$ : The net inventory level of the retailer at time point $t$

$\mathrm{IP}_{0}: \quad$ The initial inventory position of the retailer

$\operatorname{IP}(t)$ : The inventory position of the retailer at time point $t$

$u: \quad$ The mean duration of ON periods

$v: \quad$ The mean duration of OFF periods

$s: \quad$ Setup cost for each order placement

$c: \quad$ Unit product price

$h$ : $\quad$ Unit holding cost per time unit

$b_{j}$ : Unit backorder cost per time unit from customer class $i, i \in\{\mathrm{I}, \mathrm{II}\}$

$l_{i}: \quad \quad$ Unit lost sale cost from customer class $i, i \in\{\mathrm{I}, \mathrm{II}\}$

$\mathrm{BoQ}_{j}(t)$ : The backorder quantity from customer class $i$ at time point $t, i \in\{\mathrm{I}, \mathrm{II}\}$

NILS $_{i}$ : The number of lost sales from customer class $i, i \in\{\mathrm{I}, \mathrm{II}\}$

$P_{i}$ : $\quad$ The backorder probability of customer class $i, i \in\{\mathrm{I}, \mathrm{II}\}$

$q: \quad$ The proportion of customer class I

AOC: Annual ordering cost

AHC: Annual holding cost

$\mathrm{ABC}_{i}: \quad$ Annual backorder cost from customer class $i, i \in\{\mathrm{I}, \mathrm{II}\}$

ATBC: Annual total backorder cost, $\mathrm{ATBC}=\sum_{i \in\{\mathrm{I}, \mathrm{II}\}} \mathrm{ABC}_{i}$

$\mathrm{ALC}_{i}: \quad$ Annual lost sale cost from customer class $i, i \in\{\mathrm{I}, \mathrm{II}\}$

ATLC: Annual total lost sale cost, ATLC $=\sum_{i \in\{\mathrm{I}, \mathrm{II}\}} \mathrm{ALC}_{j}$

$r^{*}: \quad$ The optimal reorder point

$Q^{*}: \quad$ The optimal reorder quantity

ATC*: The minimum annual total cost.

\section{References}

[1] S. Chopra and M. S. Sodhi, "Managing risk to avoid: supplychain breakdown," MIT Sloan Management Review, vol. 46, no. 1, pp. 53-87, 2004.

[2] H. P. Chao, "Inventory policy in the presence of market disruptions," Operations Research, vol. 35, no. 2, pp. 274-281, 1987.

[3] J. S. Song and P. H. Zipkin, "Inventory control with information about supply conditions," Management Science, vol. 42, no. 10, pp. 1409-1419, 1996.

[4] B. Lewis, Inventory control with risk of major supply chain disruptions, Ph.D. thesis, Georgia Institute of Technology, 2005.

[5] M. Parlar, "Continuous-review inventory problem with random supply interruptions," European Journal of Operational Research, vol. 99, no. 2, pp. 366-385, 1997.

[6] U. Gürler and M. Parlar, "An inventory problem with two randomly available suppliers," Operations Research, vol. 45, no. 
6, pp. 904-918, 1997.

[7] A. Arreola-Risa and G. A. DeCroix, "Inventory management under random supply disruptions and partial backorders," Naval Research Logistics, vol. 45, no. 7, pp. 687-703, 1998.

[8] E. Mohebbi, "Supply interruptions in a lost-sales inventory system with random lead time," Computers and Operations Research, vol. 30, no. 3, pp. 411-426, 2003.

[9] E. Mohebbi, "A replenishment model for the supplyuncertainty problem," International Journal of Production Economics, vol. 87, no. 1, pp. 25-37, 2004.

[10] M. Parlar, Y. Wang, and Y. Gerchak, "A periodic review inventory model with Markovian supply availability," International Journal of Production Economics, vol. 42, no. 2, pp. 131-136, 1995.

[11] S. Özekici and M. Parlar, "Inventory models with unreliable suppliers in a random environment," Annals of Operations Research, vol. 91, pp. 123-236, 1999.

[12] A. Samvedi and V. Jain, "Studying the impact of various inventory policies on a supply chain with intermittent supply disruptions," in Proceedings of the Winter Simulation Conference, pp. 1641-1649, 2011.

[13] K. Moinzadeh, "Operating characteristics of the (s, S) inventory system with partial backorders and constant resupply times," Management Science, vol. 35, pp. 472-477, 1989.

[14] D. C. Montgomery, M. S. Bazaraa, and A. K. Keswani, "Inventory models with a mixture of backorders and lost sales," Naval Research Logistics, vol. 20, no. 2, pp. 255-263, 1973.

[15] D. H. Kim and K. S. Park, "(Q, r) Inventory model with a mixture of lost sales and time-weighted backorders," Journal of the Operational Research Society, vol. 36, no. 3, pp. 231-238, 1985.

[16] M. J. Posner and B. Yansouni, "A class of inventory models with customer impatience," Naval Research Logistics Quarterly, vol. 19, pp. 483-493, 1972.

[17] M. Parlar and D. Berkin, "Future supply uncertainty in EOQ models," Naval Research Logistics, vol. 38, pp. 50-55, 1991.

[18] M. Parlar and D. Perry, "Optimal (Q, r, T) policies in deterministic and random yield models with uncertain future supply," European Journal of Operational Research, vol. 84, pp. 431-443, 1993.

[19] H. J. Weiss and E. C. Rosenthal, "Optimal ordering policies when anticipating a disruption in supply or demand," European Journal of Operational Research, vol. 59, no. 3, pp. 370$382,1992$.

[20] H.-P. Chao, S. W. Chapel, C. E. Clark Jr., P. A. Morris, M. J. Sandling, and R. C. Grimes, "EPRI reduces fuel inventory costs in the electric utility industry," Interfaces, vol. 19, pp. 48-67, 1989.

[21] M. J. M. Posner and M. Berg, "Analysis of a productioninventory system with unreliable production facility," Operations Research Letters, vol. 8, no. 6, pp. 339-345, 1989.

[22] H. Groenevelt, L. Pintelon, and A. Seidmann, "Production lot sizing with machine breakdowns," Management Science, vol. 38, no. 1, pp. 104-123, 1992.

[23] X. Li and Y. Chen, "Impacts of supply disruptions and customer differentiation on a partial-backordering inventory system," Simulation Modelling Practice and Theory, vol. 18, no. 5, pp. 547-557, 2010. 

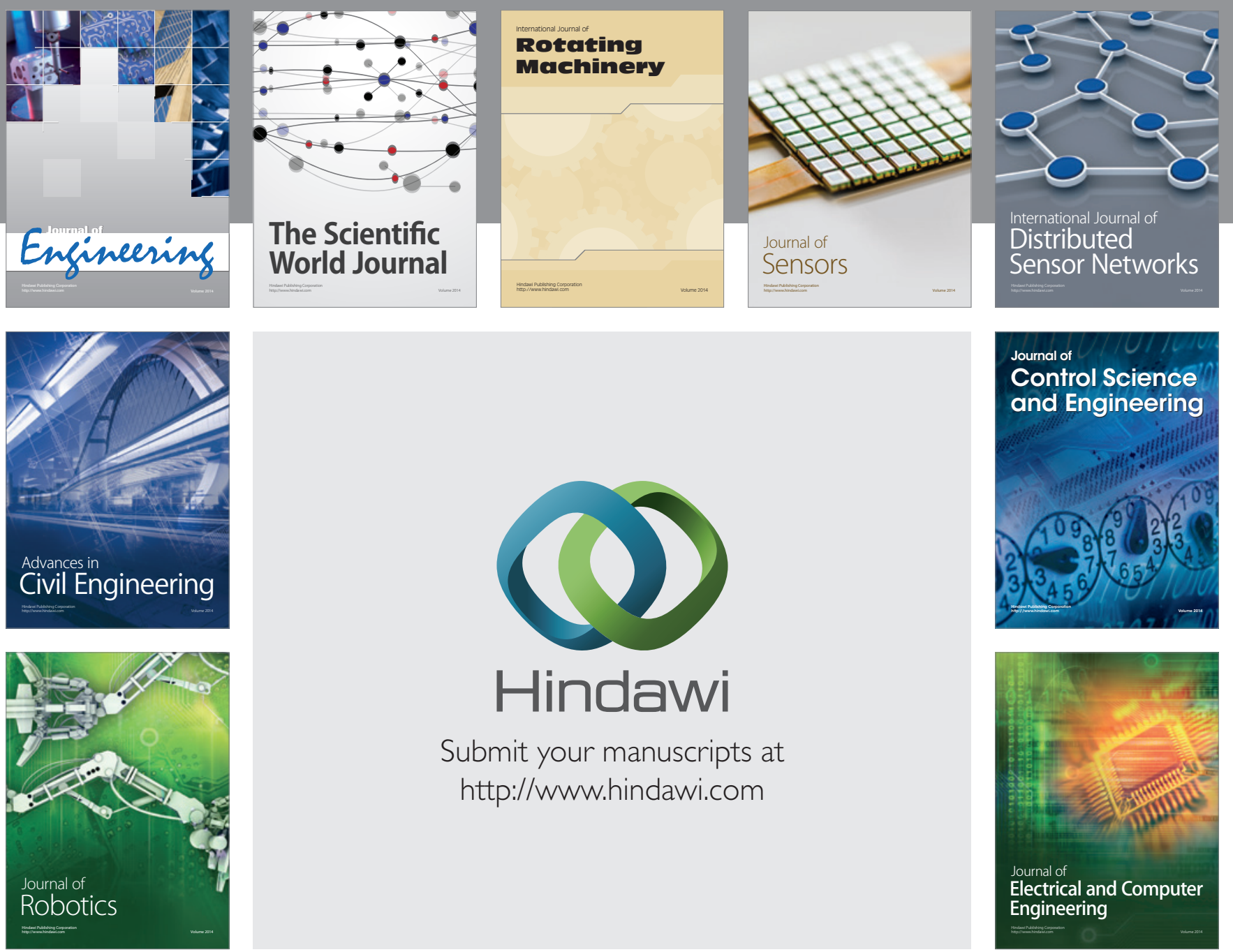

Submit your manuscripts at

http://www.hindawi.com
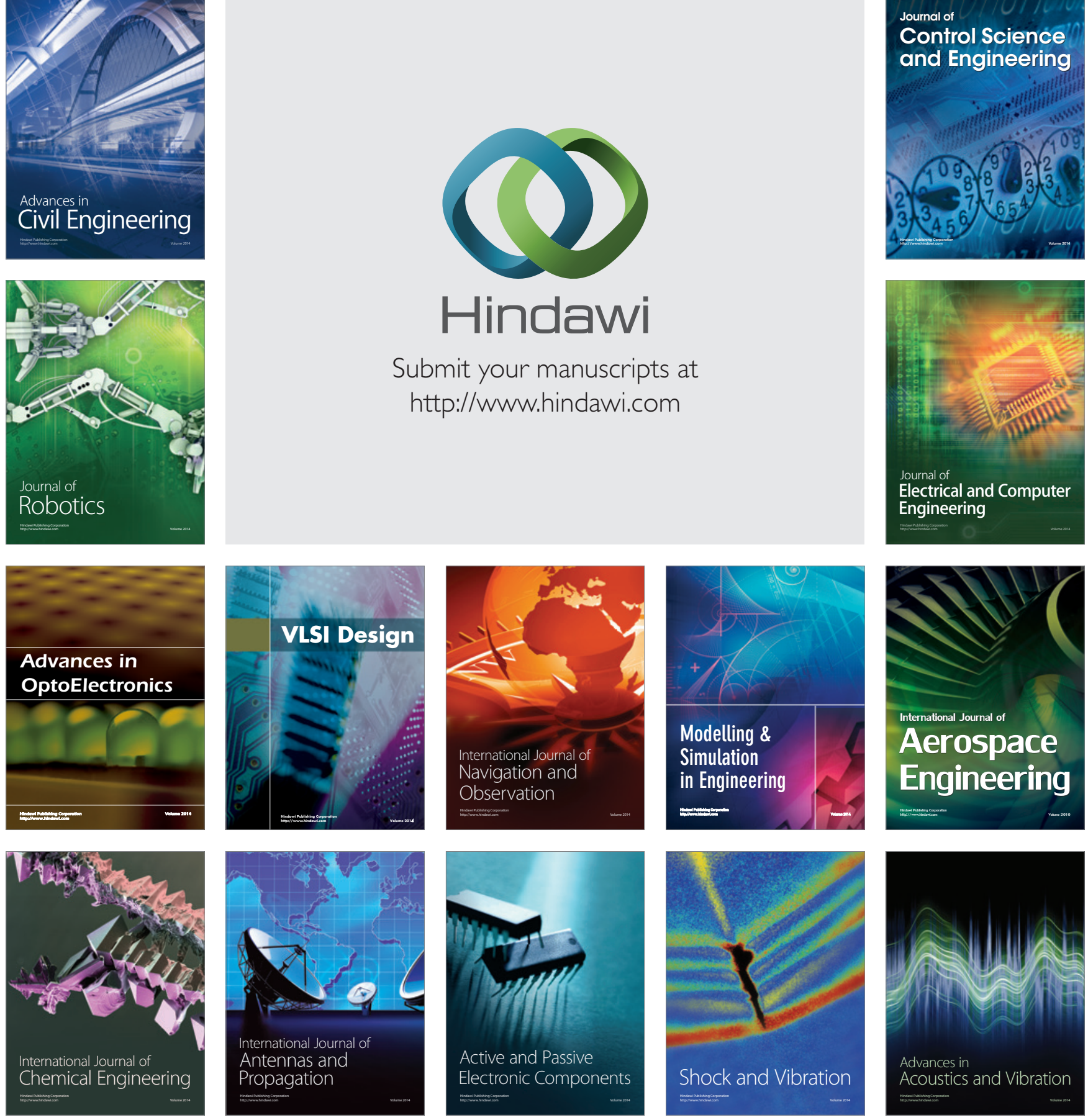